\title{
Rule Learning Based Energy Management Strategy of Fuel Cell Hybrid Vehicles Considering Multi-Objective Optimization
}

\author{
Yonggang $\mathrm{Liu}^{1 *}$, Junjun Liu${ }^{1}$, Yuanjian Zhang ${ }^{2}$, Yitao $\mathrm{Wu}^{3}$, Zheng $\mathrm{Chen}^{3,4^{*}}$ and Ming $\mathrm{Ye}^{5}$ \\ ${ }^{1}$ State Key Laboratory of Mechanical Transmissions \& School of Automotive Engineering, Chongqing University, \\ Chongqing 400044, China \\ ${ }^{2}$ Sir William Wright Technology Center, Queen's University Belfast, Belfast, BT9 5BS, United Kingdom \\ ${ }^{3}$ Faculty of Transportation Engineering, Kunming University of Science and Technology, Kunming 650500, China \\ ${ }^{4}$ School of Engineering and Materials Science, Queen Mary University of London, London, E1 4NS, United Kingdom \\ ${ }^{5}$ Key Laboratory of Advanced Manufacture Technology for Automobile Parts, Ministry of Education, Chongqing \\ University of Technology, Chongqing 400054, China \\ Correspondence: chen@kust.edu.cn (Z. Chen), and andylyg@umich.edu (Y. Liu)
}

\begin{abstract}
In this article, a multi-objective optimization-oriented energy management strategy is investigated for fuel cell hybrid vehicles on the basis of rule learning. The degradation of fuel cells and lithium-ion batteries are considered as the objective function and translated into the equivalent hydrogen consumption. The optimal fuel cell power sequence and state of charge trajectory, considered as the energy management input, are solved offline via the Pontryagin's minimum principle. The K-means algorithm is employed to hierarchically cluster the optimal data set for preparation of rules extraction, and then the rules are excavated by the improved repeated incremental pruning to production error reduction algorithm and fitted by the quasi-Newton method. The simulation results highlight that the proposed rule learning-based energy management strategy can effectively save hydrogen consumption and prolong fuel cell life with real-time application potential.
\end{abstract}

Key words: fuel cell hybrid vehicle; multi-objective optimization; energy management; rule learning.

\section{INTRODUCTION}

As a promising segment of transportation electrification, fuel cell hybrid vehicles (FCHVs) have been the research hotspot in automotive industry, owing to their zero emission, high efficiency and low noise [1]. In FCHVs, usually two energy sources including fuel cells and energy storage systems (such as lithium-ion batteries) operate together to supply the driving power. Consequently, proper power allocation between different energy sources is spurred to optimize the operation performance of FCHVs, and it is often tackled by the socalled energy management strategies (EMSs), which have been intensively investigated by industry and academia [2]. 
Generally, EMSs can be divided into rule based and optimization based strategies [3]. Rule based EMSs are usually employed for real-time application due to low computation intensity, high reliability and strong robustness; however, they still need to be optimized to further promote the vehicle overall performance. Ref. [4] proposed a rule-based meta-heuristic optimization approach for hybrid vehicles with multiple power sources. Fuzzy logic (FL) algorithms, as a special format of rule based algorithms, are introduced to control power output of energy sources [5]. Nonetheless, development of rule based strategies relies heavily on engineering experience, making it intractable to find all-sided high-quality rules for adapting to different driving conditions. In this context, optimization based EMSs progressively emerge, trying to solve the problem of energy management from optimal control perspective [6].

In general, optimization based strategies are divided into global optimization based and instantaneous optimization based algorithms. For global optimization methods, conventional solutions include dynamic programming (DP) [7] and Pontryagin's minimum principle (PMP) [8]. In [9], DP is leveraged to optimize both hydrogen consumption and batteries' serve life inside of the FCHV. In [10], the optimal hydrogen consumption of FCHV is offline attained by the PMP, and an online controller is designed according to the analysis of solved results. Ref. [11] proposes a global optimal EMS based on a multi-dimensional DP to improve the fuel economy of FCHV. In [12], DP is employed to simultaneously optimize the hydrogen economy and system durability of FCHV. A unified DP together with its effective solution is investigated to tackle the global optimal energy management of FCHV [13]. In [14], the PMP is leveraged to optimize both the fuel economy and battery lifetime. Ref. [15] applies the PMP to optimize the battery life while reducing the battery energy loss, fuel consumption and power system cost. Another kinds of global optimization methods are learning-based algorithms, such as reinforcement learning (RL) and its extensions, including Q-learning and deep RL [16]. In [17], the stationary Markov chain and RL are applied to devise the online EMS, and the simulation results suggest that the built strategy enables adaption to different driving conditions. Ref. [18] optimizes the key parameters of EMS for hybrid electric vehicles (HEVs) based on RL, and the simulation results highlight the preferable fuel economy raised by the proposed strategy. However, how to update the transition probability matrix online remains a challenging task for enhancing robustness of RL based strategy [19]. Generally, the premise of applying global optimization algorithms is that the whole driving conditions should be acquired before departure. Since driving conditions are always time-varying, it is difficult to apply global optimization methods in real-time operation. However, obtained solutions can be treated as benchmarks for evaluation of other algorithms. In addition, they 
can supply fundamental training information for artificial intelligence (AI) algorithms, like neural networks (NNs) [20]. Ref. [21] combines the speed prediction based on an adaptive online learning enhanced Markov chain and the SOC reference to distribution the driving power of FCHV. Ref. [22] utilizes the upper confidence tree search to optimize the prediction speed, and then the hierarchical RL is leveraged to achieve the approximate global optimization of FCHV. In [23], a novel $\mathrm{NN}$ is employed to dynamically regulate the equivalent factor to improve the management effect of equivalent consumption minimum strategy (ECMS). In [24], the power allocation of standard driving cycles is solved by $\mathrm{NN}$, and then driving condition identification is conducted to attain the online energy management. Ref. [25] proposes an adaptive hierarchical EMS that can be applied online for plug-in HEVs (PHEVs), and it combines deep learning and genetic algorithm to achieve power distribution between engine and battery. Actually, AI based algorithms require a variety of offline optimal data and endeavor to involve all possible situations, thereby incurring massive computation and large storage. Their controlling performance is difficult to guarantee all the time due to complicated time-varying driving conditions. In this context, instantaneous optimization-based strategies emerge, including ECMS and model predictive control (MPC) algorithms. They are declared to achieve instantaneous optimization according to current vehicle status and driving demand, together with prior management knowledge, immediate optimization and future prediction. Ref. [26] proposes a bi-level MPC algorithm to achieve the optimal torque allocation and shifting of HEVs. In [27], ECMS is implemented in a feedforward manner to systematically adjust the equivalent factor with the consideration of future driving information. In [28], a fusion algorithm combining a direct configuration method and sequential quadratic programming (SQP) is proposed to simultaneously optimize the life economy of fuel cell and energy consumption economy. One imperative premise for these instantaneous algorithms is that they need to build effective mathematical models for attaining preferable solutions [29].

As discussed above, since rule-based and optimization-based algorithms both have their intrinsic shortcomings, it comes to mind that exploring effective EMSs that can incorporate their advantages and compensate each other's defects may be a breakthrough direction. The progressively spurred rule learning principle features the advantages of less dependence on engineering experience, unnecessary requirement of prior driving conditions, simple mathematical models, acceptable interpretability and ease of extension, thus promoting its potential implementation in EMS. To the authors' knowledge, there exist only a few studies with respect to rule learning applied in energy management of FCHVs, and consequently, this motivates our in-depth research on it. Rule learning theory based on mathematical algebraic logic is mainly employed to generate rule 
sets with powerful visibility [30]. Previous researches highlight that the rule learning principle shows strong learning capability and wide adaptability in different applications [31]. One representative of rule learning algorithms is the repeated incremental pruning to produce error reduction (RIPPER) method. In [32], the RIPPER algorithm is proposed to excavate hidden rules, and the effectiveness is experimentally verified. Ref. [33] validates that the RIPPER algorithm exhibits preferable performance in view of multiclass classification. In addition, the performances of fuel cells and battery will inevitably degrade with operation, thus incurring challenges of ensuring effectiveness of EMS in the whole lifecycle of vehicle powertrain. By incorporating all the illustrated aspects, a multi-objective EMS for FCHV with real-time operation capability is herein investigated and solved by means of rule learning. To attain it, firstly, the optimal hydrogen economy that includes not only the actual consumption, but also the equivalent consumption raised by the degradation of fuel cell and battery is solved by the PMP. Next, the data set comprised of optimal solutions is hierarchically simplified using the K-means algorithm, and then the hidden rules are extracted by the RIPPER algorithm. Finally, the multi-nonlinear regression is applied to fit these rules, and the parameters are solved by the BroydenFletcher-Goldfarb-Shanno (BFGS) algorithm. The simulation results validate the effectiveness of proposed strategy in improving the hydrogen consumption economy and extending the fuel cell lifetime with online operation capability. The main contributions of this paper are attributed to the following two aspects: 1) A novel multi-objective EMS for FCHV is proposed based on the rule learning, and 2) the parameters of the fitted formulas are solved by the BFGS algorithm.

The remainder of this paper is organized as follows: In Section II, the powertrain of FCHV is introduced and modeled. In Section III, the rule learning based EMS is elaborated, including optimal data set construction and rules extraction. The simulation is carried out in Section IV, and the detailed analysis and comparison are conducted, followed by the main findings and next step work drawn in Section V.

\section{MODELING OF FCHV POWERTRAIN}

\section{A. Vehicle Model}

The powertrain structure of studied FCHV is depicted in Fig. 1. As can be seen, the fuel cell is the main source to charge the battery and drive the vehicle, and battery can supply complementary power and also recover the energy when braking. The power converter is composed of two parts: one AC/DC converter and one DC/DC converter, the $\mathrm{AC} / \mathrm{DC}$ converter performs current conversion through power factor correction, and the main 
purpose of DC/DC converter is to maintain the output voltage stable [34]. The main parameters of FCHV are listed in Table I.

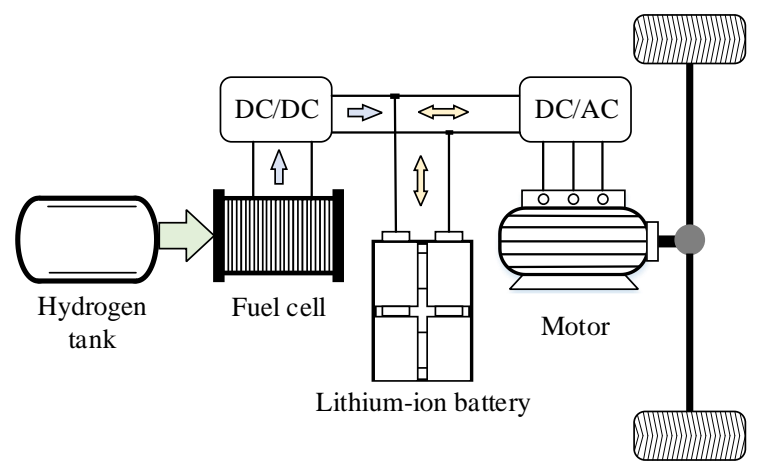

Fig. 1. Powertrain structure of FCHV.

Table I Basic parameters of FCHV

\begin{tabular}{llll}
\hline & Characteristic & Value & Unit \\
\hline \multirow{4}{*}{ Vehicle } & Mass & 1700 & $\mathrm{~kg}$ \\
parameters & Frontal projected area & 2.59 & $\mathrm{~m}^{2}$ \\
& Air drag coefficient & 0.35 & \\
& Air density & 1.29 & $\mathrm{Ns}^{2} \mathrm{~m}^{-4}$ \\
& Roll resistance coefficient & 0.014 & \\
Powertrain & Battery capacity & 5 & $\mathrm{Ah}$ \\
parameters & Battery energy & 1.5 & $\mathrm{kwh}$ \\
& Maximum power of fuel cell & 62 & $\mathrm{kw}$ \\
\hline
\end{tabular}

The total resistance when driving consists of rolling resistance, air resistance, slop resistance and acceleration resistance, as [35]:

$$
F_{t}=m g f \cos \alpha+\frac{1}{2} C_{D} A \rho V(t)^{2}+m g \sin \alpha+\delta m \frac{d V}{d t}
$$

where $F_{t}$ represents the driving force; $m, g, f$ and $\alpha$ respectively denote the total mass, gravitational acceleration, rolling resistance coefficient and road slope; $C_{D}$ is the air coefficient; $A$ is the equivalent windward area; $\rho$ represents the air density; $V$ is the real time speed, and $\delta$ is the weight coefficient of rotating mass. In this paper, the driving road is supposed with ignorable road slope and large enough adhesion, thus we can attain:

$$
F_{t}=m g f+\frac{1}{2} C_{D} \rho V(t)^{2}+\delta m \frac{d V}{d t}
$$

Now, the required driving power can be yielded, as:

$$
P_{\text {load }}(t)=V(t) \times\left\{m g f+\frac{1}{2} C_{D} \rho V(t)^{2}+\delta m \frac{d V}{d t}\right\}
$$

The bus driving power at wheels can be calculated, as:

$$
P_{r e}(t)=\frac{P_{\text {load }}(t)}{\eta_{D C / A C} \eta_{\text {motor }} \eta_{\text {trans }}}
$$


where $\eta_{D C / A C}, \eta_{\text {motor }}$ and $\eta_{\text {trans }}$ represent the efficiency of DC/AC converter, motor and transmission, respectively. The relationship between power system and demand power can be formulated, as:

$$
P_{r e}=P_{f c} \eta_{D C / D C}+P_{b}
$$

where $P_{f c}$ represents the net power of fuel cell, $P_{b}$ denotes the battery output power, and $\eta_{D C / D C}$ is efficiency of DC/DC converter. The power converter should be efficient enough to reduce the loss during power delivery. Essentially, the converter efficiency depends largely on that of the DC/AC converter [36]. However, since the research focus is the EMS in this study, which mainly manipulates the power distribution among different sources and usually does not consider much about the transient performance of powertrain components, the converter is assumed to be executed effectively, and the steady-state error and transient behavior is ignored for simplicity. In addition, the motor driving system is supposed to be able to effectively cope with voltage variations, and therefore the specific working process is not considered.

\section{B. Fuel Cell Model}

As an energy conversion device, fuel cell can convert hydrogen energy into electrical energy. The basic working principle and the efficiency-power curve of a typical fuel cell are shown in Fig. 2 (a) and (b), respectively [37]. In this study, a proton exchange membrane fuel cell (PEMFC) is deployed in the target vehicle due to its high power density and quick start-up capability. Since we focus only on the EMS in this study, and the fuel cell is considered to operate stably. The hydrogen consumption rate $\dot{m}_{H_{2}}$ can be calculated as:

$$
\dot{m}_{H_{2}}=\frac{P_{f c}}{\eta_{f c} L H V}
$$

where $\eta_{f c}$ and $L H V$ respectively denote the fuel cell efficiency and low heat value $(L H V=120 \mathrm{MJ} / \mathrm{kg})$. The hydrogen rate with respect to different powers is shown in Fig. 2 (c), which is fitted by a polynomial equation, as expressed in (7), of which the parameters are listed in Table II.

$$
\dot{m}_{H_{2}}=b_{1} \cdot P_{f c}^{4}+b_{2} \cdot P_{f c}^{3}+b_{3} \cdot P_{f c}^{2}+b_{4} \cdot P_{f c}+b_{5}
$$




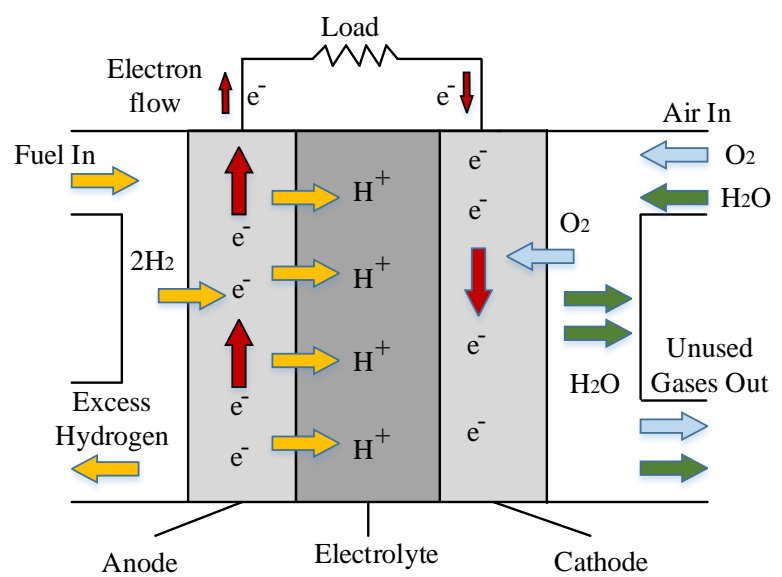

(a)

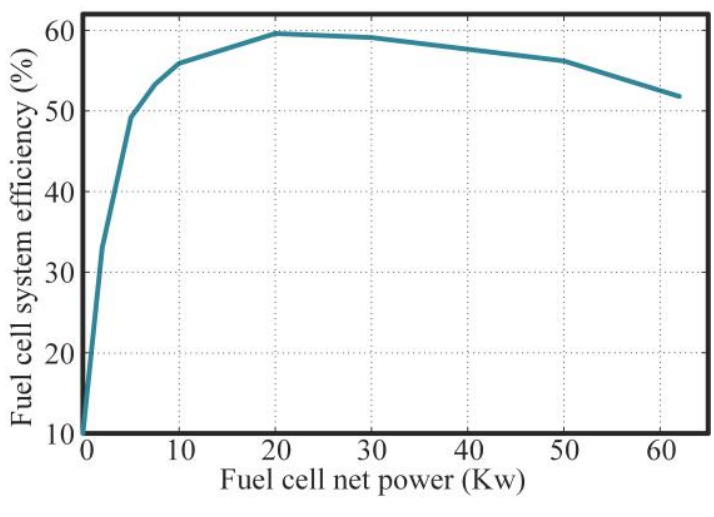

(b)

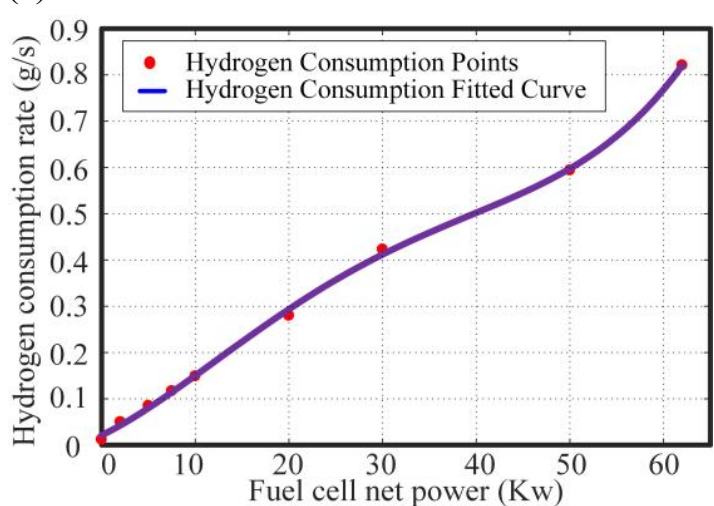

(c)

Fig. 2 The fuel cell model. (a) Basic working principle. (b) The efficiency of fuel cell. (c) The hydrogen consumption rate of fuel cell.

Table II Fitting parameters of hydrogen consumption

\begin{tabular}{ll}
\hline Fitting parameters & Value \\
\hline$b_{1}$ & $1.526 \mathrm{e}-7$ \\
$b_{2}$ & $-1.577 \mathrm{e}-5$ \\
$b_{3}$ & 0.0004387 \\
$b_{4}$ & 0.009941 \\
$b_{5}$ & 0.02112 \\
\hline
\end{tabular}

Previous researches indicate that dynamic load variation, start-stop times, idling time and heavy load can influence the lifetime of fuel cells, and the relationship is constructed by experimental validation [38], as:

$$
\Delta \phi_{F C}=k_{p}\left[\left(k_{1} t_{1}+k_{2} n_{1}+k_{3} t_{2}+k_{4} t_{3}\right)+\beta\right]
$$

where $\Delta \phi_{F C}$ is the voltage degradation percentage; $k_{p}$ is the correction factor; $t_{1}, n_{1}, t_{2}$ and $t_{3}$ represent the fuel cell's idling time, start-stop time, duration of heavy load vary and duration of heavy load, respectively; $\beta$ denotes the natural decay rate; and $k_{1}, k_{2}, k_{3}$ and $k_{4}$ are the responding degradation coefficients, as listed in Table III.

Table III Coefficients for fuel cell's performance degradation

\begin{tabular}{lll}
\hline Coefficient & Values & Definitions \\
\hline$k_{1}$ & $0.00126(\% / \mathrm{h})$ & Output power less than $2 \%$ of max power
\end{tabular}




\begin{tabular}{lll}
$k_{2}$ & $0.00196(\% / \mathrm{h})$ & $\begin{array}{l}\text { One full start-stop } \\
\text { Absolute value of load variations rate is larger } \\
k_{3}\end{array}$ \\
$k_{4}$ & $0.0000593(\% / \mathrm{h})$ & $\begin{array}{l}\text { than } 5 \% \text { of max power per second } \\
\text { Higher than } 90 \% \text { of maximal power }\end{array}$ \\
$k_{p}$ & 1.47 & \\
$\beta$ & $0.00147(\% / \mathrm{h})$ & Natural decay rate \\
\hline
\end{tabular}

The price of fuel cell stack is involved to calculate the equivalent hydrogen consumption. Generally, $10 \%$ is considered as the maximum degradation rate in automotive applications. Therefore, the equivalent hydrogen consumption raised by the voltage degradation can be calculated as:

$$
\dot{m}_{f c_{-} \text {life }}=\frac{\Delta \phi_{F C} M_{F C}}{10 \% \alpha_{H_{2}}}
$$

where $\dot{m}_{f c_{-} l i f e}$ denotes the equivalent hydrogen consumption of fuel cell degradation; $M_{F C}$ expresses the price of fuel cell, and $\alpha_{\mathrm{H}_{2}}$ represents the hydrogen price.

\section{Lithium-ion Battery Model}

The internal resistance model has been proved to be effective in characterizing the electrical performance of lithium-ion batteries [39], as shown in Fig. 3. The output power and terminal voltage of battery can be attained, as:

$$
\left\{\begin{array}{l}
P_{b}=U_{b} I_{b} \\
U_{b}=E-I_{b} R_{b}
\end{array}\right.
$$

where $E$ denotes the open circuit voltage $(\mathrm{OCV}) ; R_{b}$ is the internal resistance; and $I_{b}$ represents battery current. Furthermore, $I_{b}$ can be derived when the output power is known, as:

$$
I_{b}=\frac{E-\sqrt{E^{2}-4 R_{b} P_{b}}}{2 R_{b}}
$$

The state of charge (SOC) of battery, i.e., the ratio of the remaining capacity $Q_{\text {left }}$ over the rated value $Q$, can be calculated, as:

$$
S O C=\frac{Q_{\text {left }}}{Q}
$$

The coulomb counting algorithm expressed in (13) is applied to calculate the SOC. The resistance and voltage are considered unchanged and independent of battery SOC for simplicity [40]. 


$$
S O C(t)=S O C_{t o}-\frac{\eta_{b} \int_{t o}^{t} I_{b} d t}{3600 Q}
$$

where $\eta_{b}$ denotes the battery efficiency. During operation, the charge efficiency $\eta_{b_{-} c h r}$ and discharge efficiency $\eta_{b_{-} d i s}$ directly relate to OCV, internal resistances and output power [41], as:

$$
\left\{\begin{array}{l}
\eta_{b_{-} d i s}=\frac{1+\sqrt{1-\frac{4 R_{d i s} P_{b a t}}{E^{2}}}}{2} \\
\eta_{b_{-} c h r}=\frac{2}{1+\sqrt{1-\frac{4 R_{c h g} P_{b a t}}{E^{2}}}}
\end{array}\right.
$$

where $R_{d i s}$ and $R_{c h g}$ represent the discharge and charge resistances, respectively.

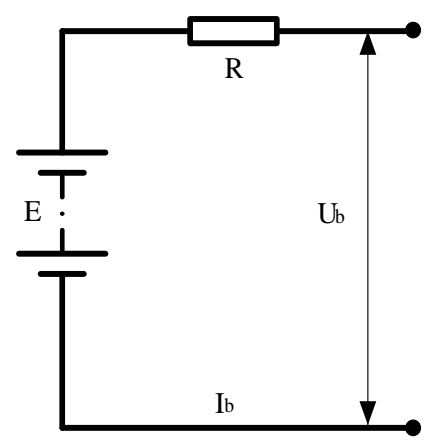

(a)

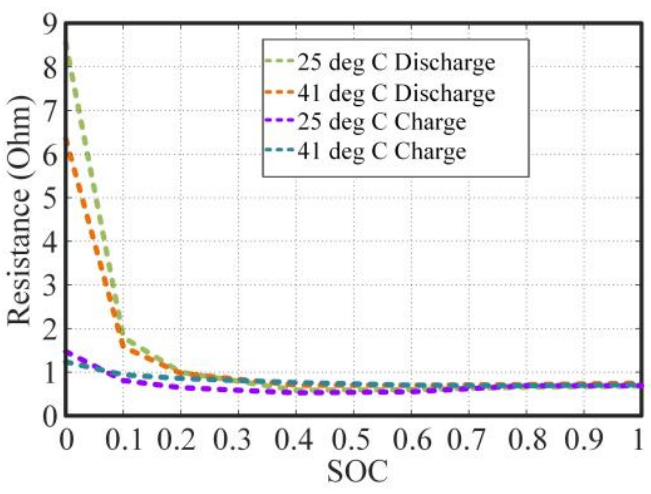

(b)

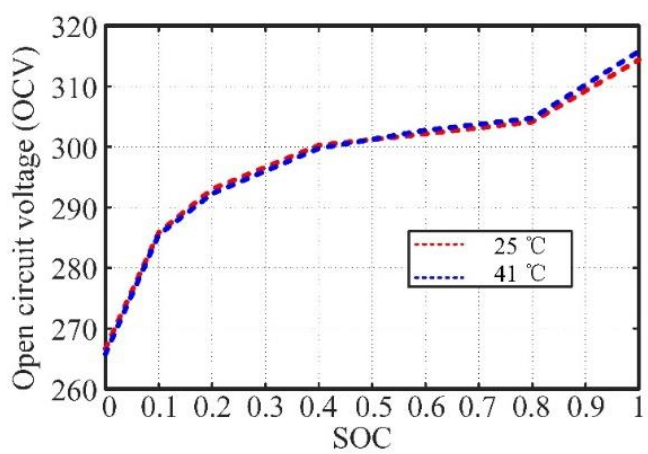

(c)

Fig. 3. Battery model and electrical performances. (a) The battery model. (b) The resistances with SOC. (c) The OCV variation with SOC.

To better evaluate the hydrogen consumption, the initial and final SOC should remain consistent in a certain trip. However, if the SOC difference exists, the equivalent hydrogen consumption should be considered, as:

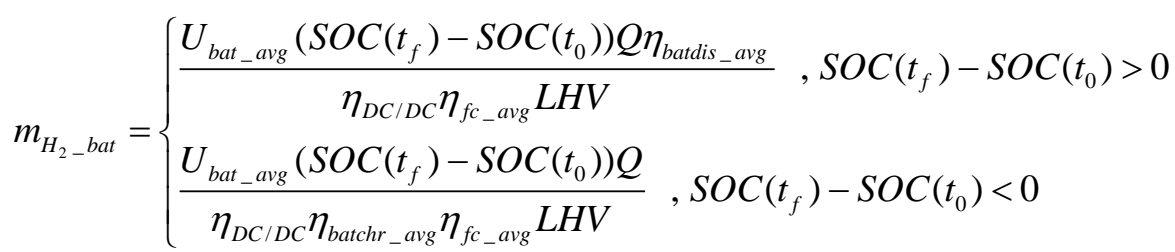


where $U_{\text {bat_avg }}$ denotes the average value of terminal voltage; $\eta_{f c_{-} a v g}$ represents the average efficiency of fuel cell; $\eta_{\text {batdis_avg }}$ and $\eta_{\text {batchr_avg }}$ denote the average discharge and charge efficiency of battery, respectively; $\operatorname{SOC}\left(t_{f}\right)$ and $\operatorname{SOC}\left(t_{0}\right)$ express the terminal and initial SOC. Previous research in [42] has indicated that the declining rate of battery capacity is related to the operating environment, activation energy, cycling times and working duration, and it can be formulated as:

$$
Q_{\text {loss }}=B \cdot \exp \left(\frac{-E_{a}}{R T}\right)\left(A_{h}\right)^{z}
$$

where $Q_{\text {loss }}$ denotes the decline percentage of battery capacity, $B$ represents the pre-exponential factor, $E_{a}$ denotes the gas activation energy, $R$ stands for gas constant, $T$ is the absolute temperature, $A_{h}$ represents the current flux, and $z$ is the power law factor. Note that equation (16) is generally employed to estimate the battery life under changeless discharge rate, and obviously, it is inappropriate to apply in a single cycle. Thus, a discrete function of battery degradation introduced in [9] is considered, as:

$$
\left\{\begin{array}{l}
\Delta Q_{\text {loss }}=\Delta B_{h} z B^{\frac{1}{z}} \exp \left(\frac{E_{a}}{z R T}\right) Q^{\frac{z-1}{z}} \\
\Delta B_{h}=\frac{1}{3600} \int_{t_{p}}^{t_{p+1}}\left|I_{b}\right| d t
\end{array}\right.
$$

Generally, in automotive applications, when the capacity decreases by $20 \%$, the battery should be abandoned and replaced. Thus, the equivalent hydrogen consumption caused by the capacity loss is taken into account, as:

$$
\dot{m}_{\text {bat_life }}=\frac{\Delta Q_{\text {loss }} M_{\text {bat }}}{20 \% \alpha_{\mathrm{H}_{2}}}
$$

where $M_{b a t}$ denote the battery price.

Now, all the related parameters that can influence the FCHV operation economy have been considered. In the next step, the EMS is constructed based on the rule learning.

\section{ENERGY MANAGEMENT STRATEGY BASED ON RULE LEARNING}

The EMS of FCHV in this research is typically a nonlinear optimization problem, of which the main optimization target includes minimization of hydrogen consumption with the consideration of degradation of different power sources, as:

$$
\min J=m_{H_{2}-b a t}+\sum\left(\dot{m}_{H_{2}}+\dot{m}_{\text {fc_life }}+\dot{m}_{\text {bat_life }}\right)
$$


As shown in Fig. 4, the whole framework of proposed EMS includes the data optimization module, simplified data module and rules extraction module, and the design process is elaborated as follows:

1) Construct a blended driving cycle with various working conditions, and find the optimal control variable sequences and corresponding state sequences;

2) Build and simplify the optimal data set;

3) Excavate the rules from the simplified data set by the rule extraction module, and apply these rules to the FCHV model;

4) Apply the rule set to different driving cycles and repeat the process from steps 1 to 3 for supplementation of the rule set.

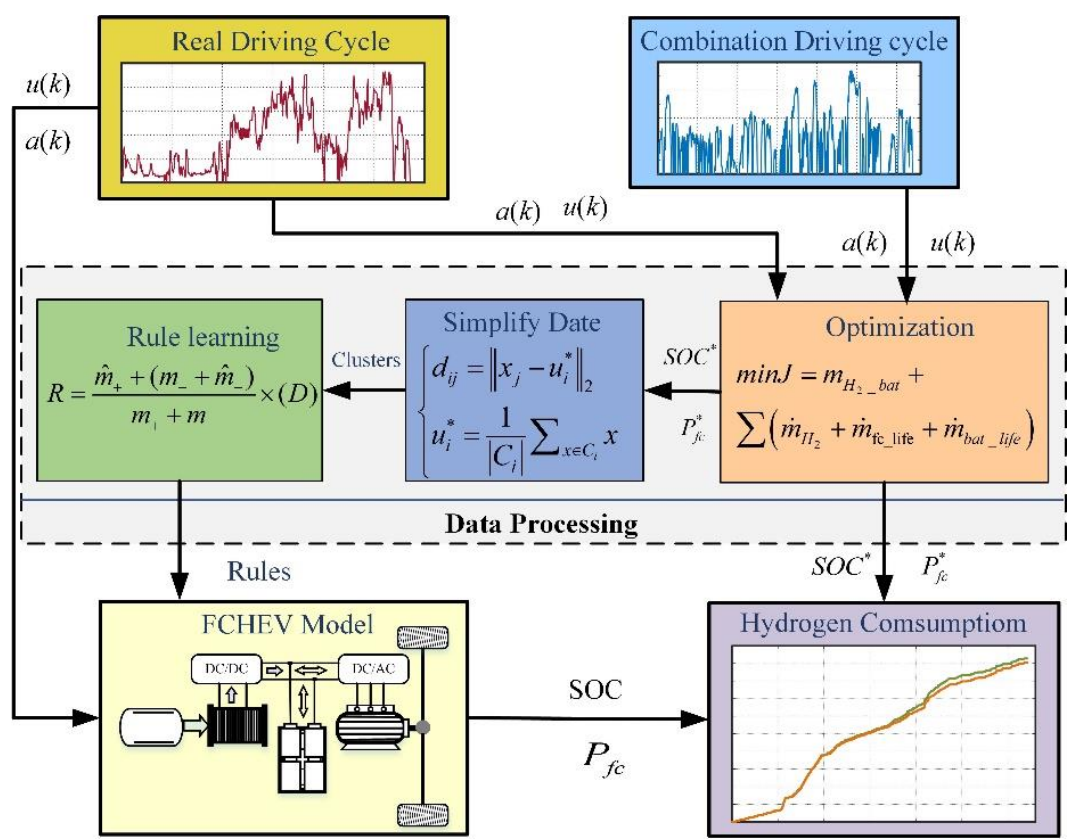

Fig. 4. Multi-objective EMS structure based on rule learning.

\section{A. Construction of Optimal Data Set}

In this study, the PMP is employed to attain the optimal control for admissible questions when the driving cycle is known [43]. During driving, the hydrogen consumption from tank as well as the equivalent hydrogen consumption incurred by the fuel cell and battery degradation constitutes the objective function, as:

$$
\left\{\begin{array}{l}
J=\Phi\left(\operatorname{SOC}\left(t_{f}\right), t_{f}\right)+\int_{t_{0}}^{t_{f}}\left(\dot{m}_{H_{2}}+\dot{m}_{f_{-} c_{-} \text {life }}+\dot{m}_{\text {bat_life }}\right) d t \\
\Phi\left(\operatorname{SOC}\left(t_{f}\right), t_{f}\right)=\left\{\begin{array}{l}
m_{H_{2} b a t}, \text { if the SOC difference meets constraint } \\
10000, \text { if the SOC difference does not meet constraint }
\end{array}\right.
\end{array}\right.
$$


where $\Phi\left(S O C\left(t_{f}\right), t_{f}\right)$ denotes the terminal cost function. During the optimization duration $\left[t_{0}, t_{f}\right], S O C$ is considered as the system state variable, and the fuel cell power is served as the control variable. The state variable and control variable should meet:

$$
S \dot{O} C(t)=-\frac{\eta_{b}}{3600 Q} \cdot \frac{E-\sqrt{E^{2}-4 R P_{b}(t)}}{2 R}
$$

and be subject to:

$$
\left\{\begin{array}{l}
\operatorname{SOC}\left(t_{0}\right)=0.6 \\
\left|\operatorname{SOC}\left(t_{f}\right)-\operatorname{SOC}\left(t_{0}\right)\right| \leq 0.005 \\
S O C_{\min } \leq S O C(t) \leq S O C_{\max }
\end{array}\right.
$$

To avoid reverse current and high load operation of fuel cell, the fuel cell power should be restricted within a certain range. Besides, the fuel cell power variation rate also needs to be constrained thanks to the limited dynamic performance of air compressor [44]. Hence, the constraints with respect to fuel cell power can be summarized, as:

$$
\left\{\begin{array}{l}
P_{f c_{\text {_min }}} \leq P_{f c_{c}}(t) \leq P_{f c_{-} \max } \\
\Delta P_{f c_{-} \text {min }} \leq \Delta P_{f c_{c}}(t) \leq \Delta P_{f c_{-} \max }
\end{array}\right.
$$

where $P_{f c_{-} \min }$ and $P_{f c_{-} \max }$ represent the minimum and maximum power of fuel cell, respectively; $\Delta P_{f c_{-} \min }$ and $\Delta P_{f c_{-} \max }$ denote the minimum and maximum fuel cell power change rate. To apply PMP [45], the Hamiltonian function is employed, as:

$$
H\left(S O C, P_{f c}, \lambda, t\right)=\dot{m}_{H_{2}}+\dot{m}_{\mathrm{fc} \_ \text {life }}+\dot{m}_{\text {bat_life }}+\lambda(t) f(x(t), u(t))
$$

where $\lambda(t)$ denotes the co-state variable. According to the conclusion of PMP, the optimal solution can be expressed as:

$$
P_{f c}^{*}=\operatorname{argmin} H\left(t, S O C^{*}(t), \lambda^{*}(t)\right)
$$

When solving the optimal control sequence [46], the following necessary conditions should be met:

1) The optimal control sequence enables minimization of the Hamiltonian function, i.e.,

$$
H\left(\operatorname{SOC}^{*}(t), P_{f c}^{*}(t), \lambda^{*}(t), t\right) \leq H\left(\operatorname{SOC}(t), P_{f c}(t), \lambda(t), t\right)
$$

2) The relationship between the co-state variable and the state variable can be formulated as: 


$$
\left\{\begin{array}{l}
\operatorname{SOC}^{*}(t)=\frac{\partial H\left(S O C^{*}(t), P_{f c}^{*}(t), \lambda^{*}(t), t\right)}{\partial \lambda(t)} \\
\dot{\lambda}^{*}(t)=-\frac{\partial H\left(S O C^{*}(t), P_{f c}^{*}(t), \lambda^{*}(t), t\right)}{\partial S O C(t)}
\end{array}\right.
$$

Then, the co-state can be solved, as:

$$
\dot{\lambda}^{*}(t)=\eta_{b} \frac{\lambda^{*}(t)}{3600 Q}\left(\frac{\partial I_{b}{ }^{*}(t)}{\partial R(t)} \frac{d R_{b}(S O C)}{d S O C}+\frac{\partial I_{b}{ }^{*}(t)}{\partial E(t)} \frac{d E(S O C)}{d S O C}\right)
$$

Since both $\lambda^{*}$ and $P_{f c}^{*}(t)$ are unknown, the difference between the initial and final SOC should be within a given range, which makes it quite difficult to be directly solved by mathematics deduction. Here, the cycle iteration is employed to find the co-state, and consequently attain the optimal control sequence. The specific process is listed as follows:

1) Initialize the co-state;

2) Discretize the control variable values within the power range of fuel cell;

3) Calculate the Hamilton function and obtain the minimum fuel cell power at the corresponding moment based on (24) and (25);

4) Apply the results in (21), and find the state variable value at this moment;

5) Repeat steps (3) and (4) and compare whether the SOC difference meets the preset threshold. If so, terminate the iteration, and select the current value as the optimal co-state value; or else, repeat steps from (1) to (4) until the difference is satisfied.

In this study, a blended driving cycle is built by combining with standard driving cycles UDDS, WVUSUB and UNIF01, and thus conventional driving conditions are all involved. The velocity, acceleration, the corresponding optimal output power of fuel cell and the SOC trajectory of battery are considered to constitute the optimal set. If the data set is directly imported to extract the rules without simplification, it will no doubt lead to huge calculation intensity during the learning process and also be prone to overfitting. To mitigate the computation intensity, the clustering method is employed to excavate the inherent properties and laws of data, thereby paving the road for further data analysis and rule abstraction. The K-means algorithm has been justified by efficient classification capabilities [47], and it is continually employed in this study. To attain it, the square error function of K-means algorithm can be formulated, as: 


$$
\begin{aligned}
E_{k}(u, C) & =\sum_{i=1}^{k} \sum_{x \in C_{i}}\left\|x-u_{i}\right\|_{2}^{2} \\
u_{i} & =\frac{1}{\left|C_{i}\right|} \sum_{x \in C_{i}} x
\end{aligned}
$$

where $E_{k}$ expresses the square error; $u$ represents the cluster center; $C$ denotes the clusters; and $x$ signifies the sample data corresponding to the cluster. The square error of each cluster can be expressed as:

$$
\min _{u} \min _{C} E(u, C)=\min _{u} \min _{C} \sum_{i=1}^{k} \sum_{x \in C_{i}}\left\|x-u_{i}\right\|_{2}^{2}
$$

Since the square error function belongs to the NP-hard problem, a heuristic iterative update method is herein adopted to resolve the minimum solution. Besides, the clustering manner is sensitive to initial cluster centers, leading to different cluster results for each run. To maintain the consistency of each clustering in all iterations, the iteration times is set to $n$ in this study. The distance sum of samples and mean vector in each cluster is calculated after each run, and then theses values will be summed. Finally, the cluster center with the minimum sum value will be taken for the final result.

To attain more accurate classification of the optimized data set, this research takes the stepwise clustering method to classify the data. The output power of fuel cell is firstly classified, followed by the velocity and acceleration, and finally the SOC of auxiliary power source is classified. The output power of fuel cell is divided into three categories based on its characteristics, and according to the driving cycle features, both the velocity and acceleration are divided into four types, and the SOC is classified into three classes. After completing the hierarchical clustering process, the median of each cluster is exploited to finally determine its category, and the classification with less data will be removed, thereby laying the foundation for rules extraction.

\section{B. Rules Extraction}

This study applies the rule learning to excavate the hidden rules from the simplified data set. The goal is to construct a rule set trying to involve as many samples as possible. Actually, the traditional RIPPER algorithm shows strong capabilities of generalization performance and fast learning speed, except the inadequate rule covering problem [48]. To cope with it, an improved RIPPER algorithm is proposed, endeavoring to summarize as many rules as possible. After finishing the extraction of one rule, the data included by the new rule set will be eliminated, and the next excavation is performed until no new rule can be found. The rules will be prioritized according to the order of learned rules. Note that in each rule learning process, the learned rules with $100 \%$ 
coverage rate will be firstly added in the rule set. When no new rules can be extracted according to the evaluation criterion, the rules with higher accuracy will be imported, and then when the rules' accuracy is the same, the rule including more samples will be written into the rule set. The process of the improved RIPPER algorithm can be summarized in Table IV, and the performance metrics of the algorithm can be expressed as:

$$
M=\frac{\hat{m}_{+}+\left(m_{-}+\hat{m}_{-}\right)}{m_{+}+m_{-}}
$$

where $M$ denotes the performance index; $\hat{m}_{+}$and $\hat{m}_{-}$represent the number of positive and negative examples covered by the rule, respectively; $m_{+}$and $m_{-}$is the amount of positive and negative examples in the data set. As can be found, three times rules' extraction is conducted via the algorithm, and a total of fourteen specific rules are derived, as listed in Table V. Fig. 5 depicts the learned rules in three dimensions, and obviously no overlap exists. Nonetheless, the rule set may not involve all the situations; therefore, a default rule needs to be set to deal with the uncovered samples. The default rule is formulated on the basis of deterministic rule.

Table IV Improved RIPPER Algorithm

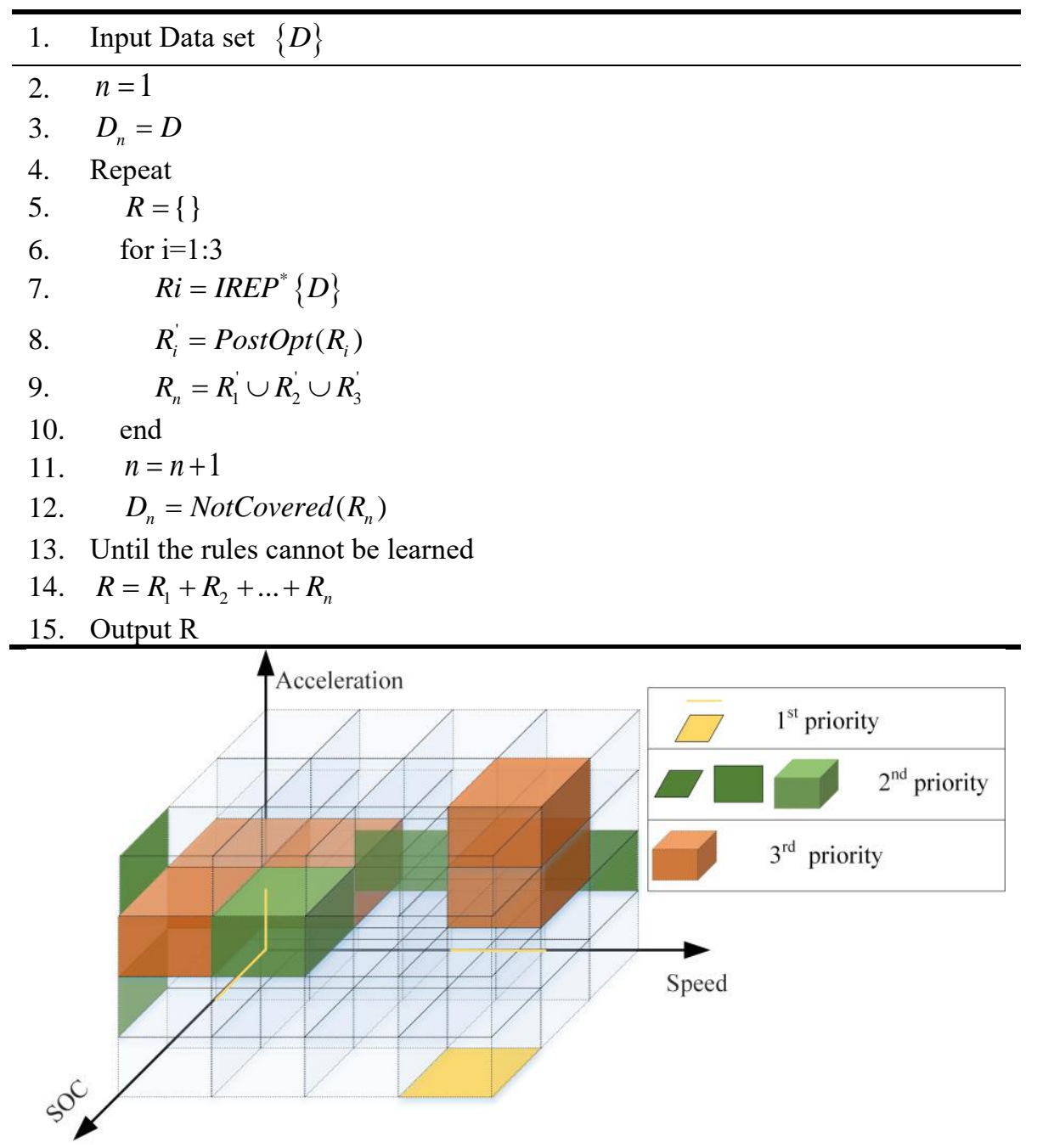

Fig. 5. Rule visualization. 
Table V Learned rule set

\begin{tabular}{llllll}
\hline & Speed & Acceleration & SOC & Category & Order \\
\hline 1 & $/$ & Urgent deceleration & $/$ & First & $1^{\text {st }}$ \\
2 & $/$ & $/$ & Low & First & $1^{\text {st }}$ \\
3 & Fairly high & $/$ & $/$ & Second & $1^{\text {st }}$ \\
4 & High & $/$ & High & Third & $1^{\text {st }}$ \\
5 & $/$ & Urgent acceleration & High & First & $2^{\text {nd }}$ \\
6 & Medium & Deceleration & $/$ & First & $2^{\text {nd }}$ \\
7 & $/$ & Deceleration & High & First & $2^{\text {nd }}$ \\
8 & High & Deceleration & $/$ & Second & $2^{\text {nd }}$ \\
9 & Medium & Acceleration & High & Third & $2^{\text {nd }}$ \\
10 & Low & Acceleration & Medium & First & $3^{\text {rd }}$ \\
11 & Low & Acceleration & High & First & $3^{\text {rd }}$ \\
12 & Medium & Acceleration & Medium & Third & $3^{\text {rd }}$ \\
13 & High & Acceleration & Medium & Second & $3^{\text {rd }}$ \\
14 & High & Urgent acceleration & Medium & First & $3^{\text {rd }}$ \\
\hline
\end{tabular}

To promote the inclusiveness of rules and reduce the complexity of choosing data, a nonlinear regression is used to fit the rules' data. As one of the most popular manners, the quasi-Newton method is qualified in solving nonlinear optimization problems, and the BFGS algorithm is introduced in this study [49]. To make the algorithm more general, this study considers the situation of multiple inputs. First, the fuel cell power of fuel cell is considered as the objective function $f(x)$, and then the second-order Taylor expansion is introduced, as:

$$
\Psi(x)=f\left(x_{k}\right)+\nabla f\left(x_{k}\right)\left(x-x_{k}\right)+\frac{1}{2}\left(x-x_{k}\right)^{T} \nabla^{2} f\left(x_{k}\right)\left(x-x_{k}\right)
$$

where $x=\left(x_{1}, x_{2}, \ldots, x_{N}\right) \in R^{N}, x_{k}$ is the estimated value of current minimum, and $\nabla f$ and $\nabla^{2} f$ denoting the gradient vector and Hessian matrix of $f$ can be defined as:

$$
\left\{\begin{array}{c}
\nabla f=\left[\frac{\partial f}{\partial x_{1}}, \frac{\partial f}{\partial x_{2}}, \ldots, \frac{\partial f}{\partial x_{N}}\right]^{T} \\
\nabla^{2} f=\left[\begin{array}{cccc}
\frac{\partial^{2} f}{\partial x_{1}^{2}} & \frac{\partial^{2} f}{\partial x_{1} \partial x_{2}} & \cdots & \frac{\partial^{2} f}{\partial x_{1} \partial x_{N}} ; \\
\frac{\partial^{2} f}{\partial x_{2} \partial x_{1}} & \frac{\partial^{2} f}{\partial x_{2}^{2}} & \cdots & \frac{\partial^{2} f}{\partial x_{2} \partial x_{N}} ; \\
\vdots & \vdots & \ddots & \vdots \\
\frac{\partial^{2} f}{\partial x_{N} \partial x_{1}} & \frac{\partial^{2} f}{\partial x_{N} \partial x_{2}} & \ldots & \frac{\partial^{2} f}{\partial x_{N}^{2}}
\end{array}\right]_{N \times N}
\end{array}\right.
$$

Here, let $g_{k}=\nabla f\left(x_{k}\right)$ and $H_{k}=\nabla^{2} f\left(x_{k}\right)$. The iteration equation can be derived, as:

$$
x_{k+1}=x_{k}-H_{k}^{-1} g_{k}, \quad k=0,1, \ldots
$$

Therefore, the search direction is $d_{k}=-H_{k}^{-1} g_{k}$. Here, the Quasi-Newton function is firstly provided before detailing the BFGS algorithm. To this end, a positive definite matrix is constructed to replace $H_{k}$ and mitigate 
the large computation complexity when solving second-order partial derivatives. By supposing $x_{k+1}$ can be obtained after $k+1$ iterations, the objective function can be expanded according to the two-order Taylor equation in the vicinity of $x_{k+1}$, as:

$$
f(x) \approx f\left(x_{k+1}\right)+\nabla f\left(x_{k+1}\right)\left(x-x_{k+1}\right)+\frac{1}{2}\left(x-x_{k+1}\right)^{T} \nabla^{2} f\left(x_{k+1}\right)\left(x-x_{k+1}\right)
$$

The gradient operation is imposed to both sides of the above equation, and thus:

$$
\nabla f(x) \approx \nabla f\left(x_{k+1}\right)+H_{k+1}\left(x-x_{k+1}\right)
$$

Let $x=x_{k}, s_{k}=x_{k+1}-x_{k}, \quad y_{k}=g_{k+1}-g_{k}$, and summarize the above equations to derive the Quasi-Newton function, as:

$$
y_{k} \approx H_{k+1} s_{k}
$$

Then, $H_{k+1}$ is replaced with $B_{k+1}$ during the iteration, i.e., $H_{k+1} \approx B_{k+1}$. Here, $B$ is considered to represent the approximation of the Hessian matrix. The core of BFGS algorithm is to solve $B_{k+1}$ by the iterative method, which can be formulated as:

$$
B_{k+1}=B_{k}+\Delta B_{k}, \quad k=0,1,2, \ldots
$$

where $B_{0}$ denotes the identity matrix, and the correction matrix is constructed into the following equation:

$$
\Delta B_{k}=\alpha u u^{T}+\beta v v^{T}
$$

where $\alpha$ and $\beta$ are the undetermined coefficients, and $u, v \in \mathbb{R}^{N}$ denote the undetermined vectors. By combining (38) and (39), we can attain:

$$
y_{k}=B_{k} s_{k}+\left(\alpha u^{T} s_{k}\right) u+\left(\beta v^{T} s_{k}\right) v
$$

Obviously, both $\alpha u^{T} s_{k}$ and $\beta v^{T} s_{k}$ are real numbers. Let $\alpha u^{T} s_{k}=1$ and $\beta v^{T} s_{k}=-1$, then

$$
y_{k}=B_{k} s_{k}+u-v
$$

To ensure that equation (42) is true, we assume that $u=y_{k}$ and $v=B_{k} s_{k}$, and then the correction matrix $\Delta B_{k}$ can be obtained, as:

$$
\Delta B_{k}=\frac{y_{k} y_{k}^{T}}{y_{k}^{T} s_{k}}-\frac{B_{k} s_{k} s_{k}^{T} B_{k}}{s_{k}^{T} B_{k} s_{k}}
$$

By this manner, the BFGS algorithm can be effectively applied to the rule fitting and validation. Additionally, the root-mean-square error (RMSE) is employed in this study to evaluate the nonlinear fitting performance, as: 


$$
R M S E=\sqrt{\frac{1}{m} \sum_{i=1}^{m}\left(y_{i}-\hat{y}_{i}\right)^{2}}
$$

where $m$ is sample number; $y_{i}$ and $\hat{y}_{i}$ respectively denote sample value and fitting function output value.

By means of the previous processes, the rules can be extracted and fitted. In the next step, the simulations are conducted to validate the feasibility of proposed algorithm.

\section{SIMULATION AND DISCUSSION}

To validate the effectiveness of the multi-objective EMS based on rule learning, simulations were executed under Matlab/Simulink on a desktop computer with an i5 processor and 8 gigabyte memories. The simulation results of proposed strategy are compared with those based on PMP and deterministic rule-based algorithms. In this study, the rule-based EMS is described as follows. When the demand power is less than $5 \mathrm{Kw}$, the fuel cell does not turn on; and the net power of fuel cell should meet the required power when the power demand is in the high efficiency zone ( 5 to $17 \mathrm{Kw}$ ). When the propelling power is high, the fuel cell only provides the constant power. According to the discussion, the objective and constraints of variables can be expressed as:

$$
J=m_{H_{2}-b a t}+\int_{t_{0}}^{t_{f}}\left(\dot{m}_{H_{2}}+\dot{m}_{\text {fc_life }}+\dot{m}_{\text {bat_life }}\right) d t
$$

subject to:

$$
\left\{\begin{array}{l}
P_{f c}(t)-P_{f c_{-} \min } \geq 0 \\
P_{f c_{-} \max }-P_{f c}(t) \geq 0 \\
P_{f c}(t)-P_{f c}(t-1)-\Delta P_{f c_{-} \min } \geq 0 \\
\Delta P_{f c_{-} \max }-P_{f c}(t)+P_{f c}(t-1) \geq 0 \\
S O C(t)-S O C_{\min } \geq 0 \\
S O C_{\max }-S O C(t) \geq 0
\end{array}\right.
$$

where $\Delta P_{f c_{-} \min }=-5 \mathrm{Kw}$, and $\Delta P_{f c_{-} \max }=5 \mathrm{Kw}$. Note that when the required power exceeds the maximum capability of fuel cell and battery, the dynamic performance of air compressor is supposed to be qualified in a short time to meet the power demand. The simulation validations were conducted under the LA92, WLTC, WVUCITY and INDIA_URBAN cycles, and the results are listed in Table VI. As can be seen, the optimization effects based on the deterministic rule strategy is greatly related to the driving condition. However, the hydrogen consumption based on the rule learning strategy is closer to PMP. The data in Table VI is split into the hydrogen consumed from the tank during driving and equivalent hydrogen consumption of fuel cell and battery 
degradation, as listed in Table VII. Apparently, the fuel cell degradation rate based on the deterministic rulebased method is faster than that of other two strategies due to the limited conditions of triggering the fuel cell's operation.

Table VI Hydrogen consumption

\begin{tabular}{llll}
\hline \multirow{2}{*}{ Driving Cycle } & \multicolumn{3}{c}{ Hydrogen consumption $(\mathrm{g})$} \\
\cline { 2 - 4 } & Rule-based & PMP-based & Rule learning-based \\
\hline \multirow{2}{*}{ LA92 } & 3786.3284 & 3633.7976 & 3760.766 \\
\multirow{2}{*}{ WLTC } & $+0.68 \%$ & $-3.38 \%$ & \\
& 4634.9575 & 4352.3835 & 4606.5069 \\
WVUCITY & $+0.62 \%$ & $-5.51 \%$ & \\
& 3369.4657 & 3149.3213 & 3180.5627 \\
HWFET & $+5.94 \%$ & $-0.98 \%$ & \\
& 1812.6884 & 1813.0085 & 1949.8294 \\
INDIA_URBAN_SAMPLE & $-7.03 \%$ & $-7.01 \%$ & \\
& 6514.7812 & 6120.6878 & 6287.0687 \\
\hline
\end{tabular}

Table VII Distribution of hydrogen consumption

\begin{tabular}{|c|c|c|c|c|}
\hline \multirow[b]{2}{*}{ Driving cycle } & \multirow[b]{2}{*}{ Algorithms } & \multicolumn{3}{|c|}{ Hydrogen consumption } \\
\hline & & $\begin{array}{l}\text { Hydrogen tank } \\
\operatorname{cost}(\mathrm{g})\end{array}$ & $\begin{array}{l}\text { Fuel cell } \\
\text { degradation }(\mathrm{g})\end{array}$ & $\begin{array}{l}\text { Battery degradation } \\
(\mathrm{g})\end{array}$ \\
\hline \multirow{5}{*}{ LA92 } & \multirow{2}{*}{ Rule-based } & 149.7311 & 3171.6953 & 464.9021 \\
\hline & & $+1.50 \%$ & $+7.89 \%$ & $-30.98 \%$ \\
\hline & \multirow{2}{*}{ PMP-based } & 134.9291 & 2928.7735 & 570.095 \\
\hline & & $-8.53 \%$ & $-0.372 \%$ & $-15.36 \%$ \\
\hline & Rule learning-based & 147.5227 & 2939.6997 & 673.5436 \\
\hline \multirow{5}{*}{ WLTC } & \multirow{2}{*}{ Rule-based } & 204.4405 & 3942.9177 & 487.5993 \\
\hline & & $-10.35 \%$ & $+6.84 \%$ & $-29.28 \%$ \\
\hline & \multirow{2}{*}{ PMP-based } & 214.4563 & 3671.0632 & 466.864 \\
\hline & & $-5.37 \%$ & $-0.52 \%$ & $-32.28 \%$ \\
\hline & Rule learning-based & 226.6368 & 3690.4255 & 689.4447 \\
\hline \multirow{5}{*}{ HWFET } & \multirow{2}{*}{ Rule-based } & 138.6455 & 1597.9558 & 76.087 \\
\hline & & $-11.26 \%$ & $+2.16 \%$ & $-66.83 \%$ \\
\hline & \multirow{2}{*}{ PMP-based } & 139.4458 & 1562.4709 & 111.0918 \\
\hline & & $-10.74 \%$ & $-0.11 \%$ & $-51.57 \%$ \\
\hline & Rule learning-based & 156.231 & 1564.2273 & 229.3711 \\
\hline \multirow{5}{*}{ WVUCITY } & \multirow{2}{*}{ Rule-based } & 52.2974 & 3204.4095 & 112.588 \\
\hline & & $-12.58 \%$ & $+9.61 \%$ & $-42.92 \%$ \\
\hline & \multirow{2}{*}{ PMP-based } & 48.782 & 2871.6743 & 228.865 \\
\hline & & $-18.45 \%$ & $-1.77 \%$ & $+16.035 \%$ \\
\hline & Rule learning-based & 59.82 & 2923.5052 & 197.2374 \\
\hline \multirow{5}{*}{$\begin{array}{l}\text { INDIA_URBAN } \\
\text { SAMPLE }\end{array}$} & \multirow{2}{*}{ Rule-based } & 141.3173 & 6055.7546 & 317.7093 \\
\hline & & $+1.25 \%$ & $+7.54 \%$ & $-38.47 \%$ \\
\hline & \multirow{2}{*}{ PMP-based } & 118.8226 & 5486.0024 & 515.8628 \\
\hline & & $-14.86 \%$ & $-2.58 \%$ & $-0.099 \%$ \\
\hline & Rule learning-based & 139.5664 & 5631.1278 & 516.3744 \\
\hline
\end{tabular}

Next, the above strategies are implemented in the NEDC cycle, and the speed trajectory and the corresponding demand power is depicted in Fig. 6 (a). The initial SOC is set to 0.6, and the SOC trajectories are described in Fig. 6 (b). An iterative method is adopted in the PMP to choose the proper co-state value for regulating the fuel cell power until the difference between the initial and final SOC maintains within a preset boundary; and the proposed strategy and rule-based strategy determine the fuel cell power according to the 
vehicle current state, which makes the difference of SOC more obvious. The hydrogen consumption is listed in Table VIII. As can be seen, the deterministic rule-based strategy costs $4.59 \%$ more hydrogen than the proposed strategy, while the PMP consumes 3.24\% less than the proposed strategy, indicating that the proposed strategy leads to better fuel economy than the rule-based strategy, and the optimization effect is close to the global optimal result.

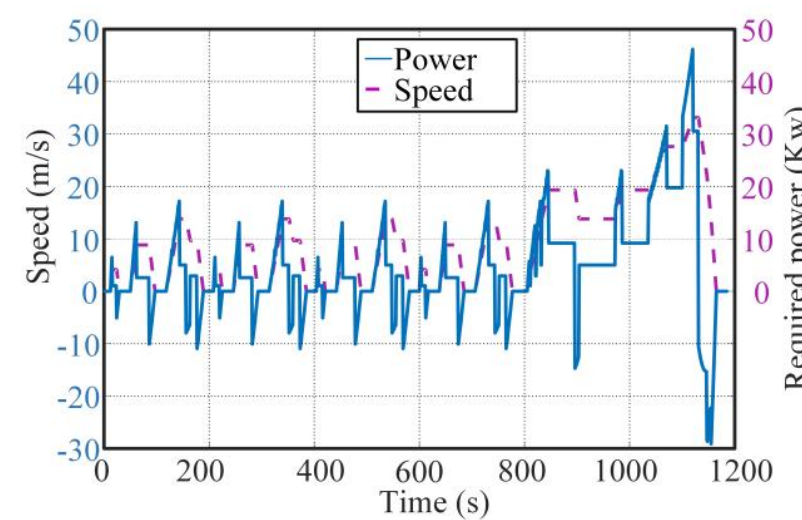

(a)

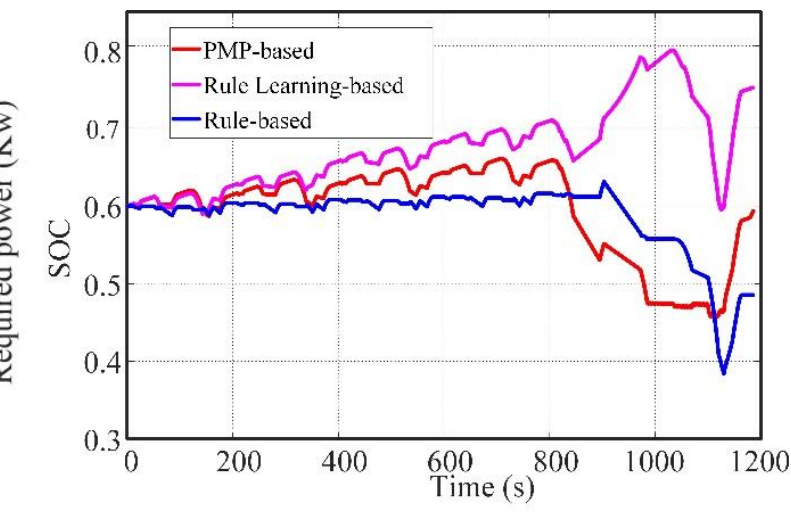

(b)

Fig. 6. NEDC driving cycle. (a) The trajectories of speed and power demand. (b) SOC trajectories.

Table VIII Hydrogen consumption with various strategies

\begin{tabular}{|c|c|c|c|c|}
\hline & $\begin{array}{l}\text { Hydrogen consumption } \\
\text { during driving }(\mathrm{g})\end{array}$ & $\triangle S O C$ & $\begin{array}{ll}\text { Equivalent } & \text { hydrogen } \\
\text { consumption of } & \triangle S O C(\mathrm{~g})\end{array}$ & $\begin{array}{l}\text { Total hydrogen } \\
\text { consumption }(\mathrm{g})\end{array}$ \\
\hline Rule-based & $\begin{array}{l}2929.5133 \\
(+90.9013 g)\end{array}$ & -0.11 & -21.1429 & $\begin{array}{l}2950.6562 \\
(+4.59 \%)\end{array}$ \\
\hline PMP-based & $\begin{array}{l}2727.7021 \\
(-110.9099 g)\end{array}$ & -0.005 & -1.773 & $\begin{array}{l}2729.4751 \\
(-3.24 \%)\end{array}$ \\
\hline Rule learning based & 2838.612 & 0.15 & 17.5286 & 2821.0834 \\
\hline
\end{tabular}

Note: + Increment; -: Decrement

Based on the three strategies, the power variation of fuel cell and the distribution of operating points are depicted in Figs. 7 and 8, respectively. Obviously, the PMP strategy enables higher operating efficiency of fuel cell to minimize the hydrogen consumption, and the other two strategies are only relevant with the current required power. The PMP based algorithm regulates the fuel cell power most frequently, and the rule learning based strategy can switch the rules according to the different driving conditions, therefore the power is more concentrated in the high efficiency region. Moreover, since the rule-based strategy enables the fuel cell to operate only in the high efficiency zone, the power variation range is the least. 


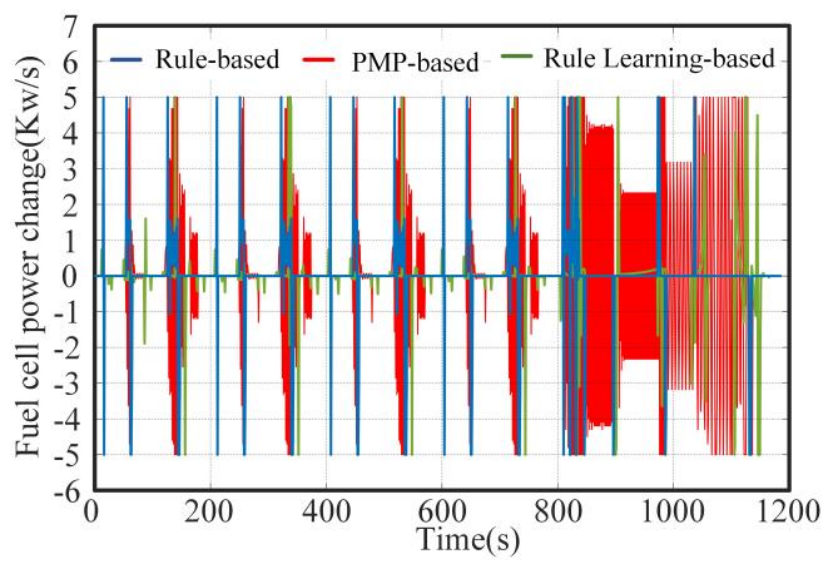

Fig. 7. Power variation of fuel cell.

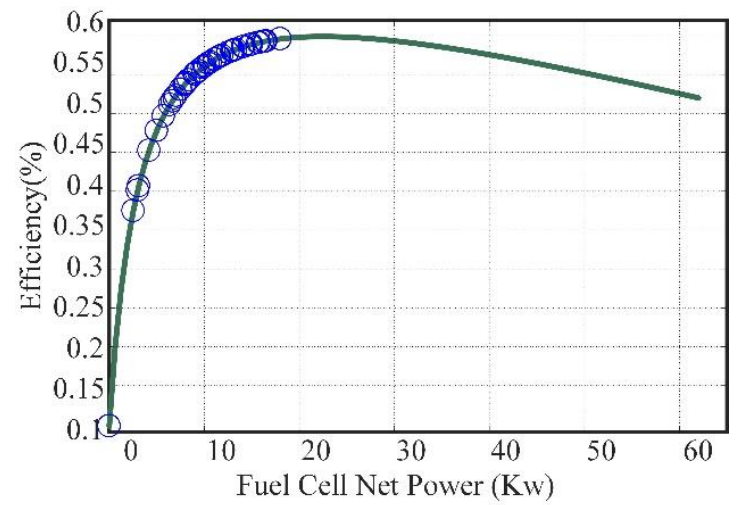

(a)

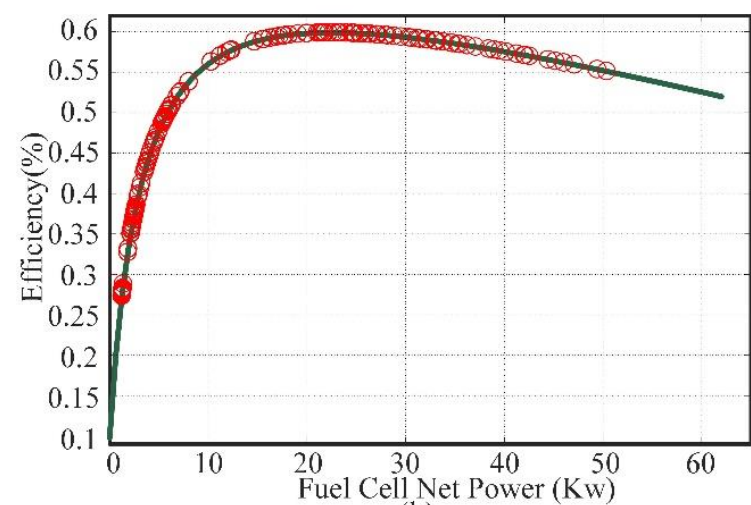

(b)

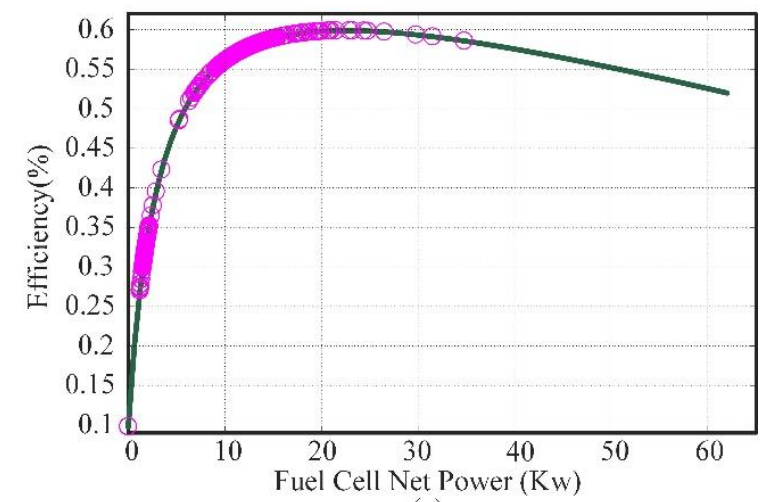

(c)

Fig. 8. Net power working points of fuel cell. (a) Rule-based method. (b) PMP-based method. (c) Rule learningbased method.

To further evaluate the fuel cell and battery degradation performance in terms of three strategies, the degradation related hydrogen consumption is compared in Fig. 9, where (a) exhibits the total hydrogen consumption during the trip. Obviously, the equivalent hydrogen consumption needs to be recalculated due to the nonzero SOC difference, and the results are listed in Table IX. Obviously, the proposed strategy in this paper consumes the least hydrogen during the trip. Fig. 9 (b) is the total equivalent hydrogen consumption caused by the fuel cell degradation. The equivalent hydrogen consumption by three strategies shows a linear increasing trend due to the high cost of fuel cell. The strategy based on the deterministic rules does not take the fuel cell's 
degradation into account, thereby resulting in faster degradation speed, while the degradation speeds by the other two strategies are basically the same. Fig. 9 (c) depicts the variation of equivalent hydrogen consumption caused by the battery degradation. From (17), the fluctuation of battery current is positively related to its capacity degradation. The battery current of the rule-based strategy does not change obviously, leading to the lowest equivalent hydrogen consumption. The equivalent hydrogen consumption of battery degradation by the PMP-based strategy is in between due to the pursuit of high efficiency, and the rule learning-based strategy cost mostly due to the frequent switched rules.

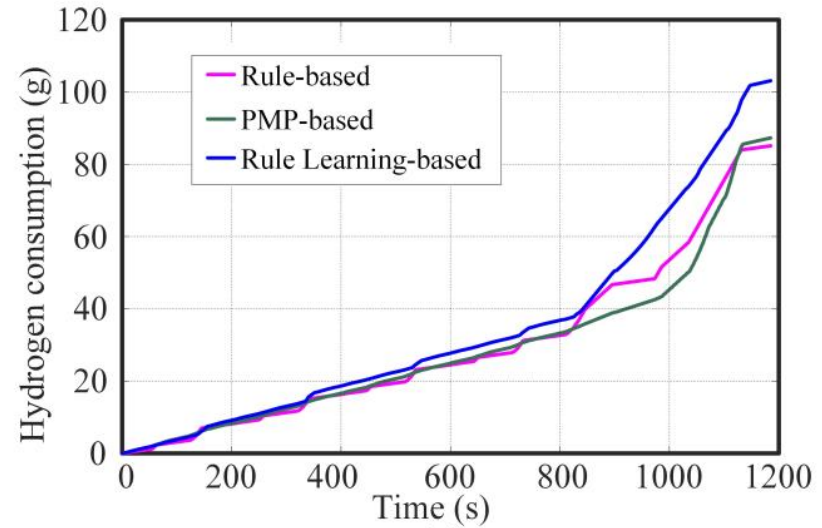

(a)

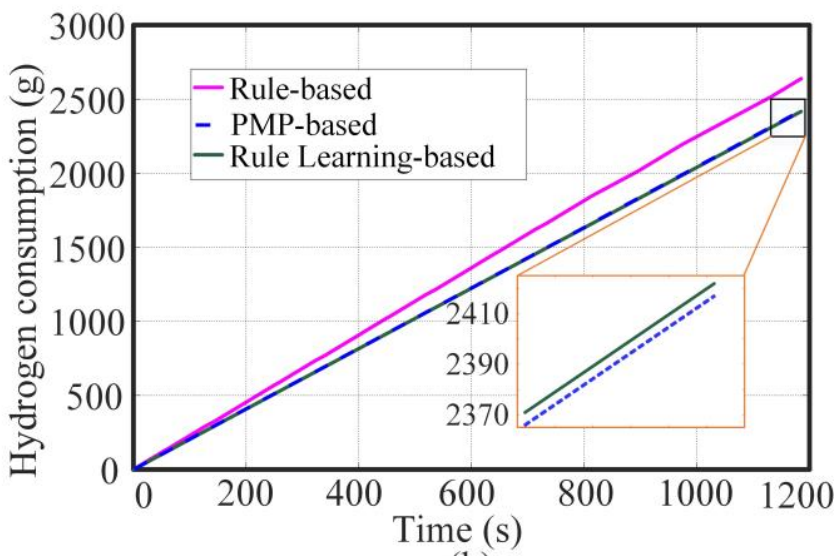

(b)

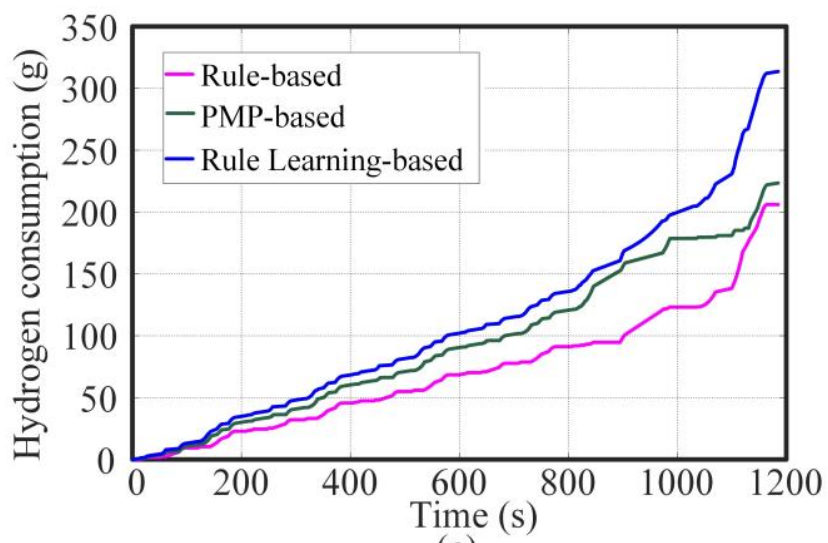

(c)

Fig. 9. Hydrogen consumption. (a) Direct hydrogen consumption of fuel cell. (b) Equivalent hydrogen consumption of fuel cell. (c) Equivalent hydrogen consumption of battery.

Table IX Hydrogen consumption from hydrogen tank

\begin{tabular}{llll}
\hline & $\begin{array}{l}\text { Hydrogen consumption from } \\
\text { hydrogen tank }(\mathrm{g})\end{array}$ & $\begin{array}{l}\text { Hydrogen consumption of } \\
\text { charging or discharging for } \\
\text { battery }(\mathrm{g})\end{array}$ & $\begin{array}{l}\text { Total hydrogen } \\
\text { consumption }(\mathrm{g})\end{array}$ \\
\hline Rule-based & 85.124 & -21.1429 & $\begin{array}{l}106.2669 \\
+24.14 \%\end{array}$ \\
PMP-based & 87.3196 & -1.773 & 89.0926 \\
Rule learning-based & 103.1289 & 17.5286 & $85.08 \%$ \\
\hline
\end{tabular}

The simulation results indicate that the proposed strategy sacrifices the battery lifetime to prolong the fuel cell's lifetime, and it is reasonable from the economic cost perspective. The equivalent hydrogen consumption 
of power sources degradation is shown in Table X. To verify the online application potential of the proposed strategy, the calculation intensity based on different algorithms is investigated, as compared in Table XI. As can be found, the single-step calculation duration of three strategies is all less than $0.4 \mathrm{~ms}$. However, the PMP strategy needs to know global operating conditions in advance, thus hindering its online application potential. Obviously, the single-step calculation time of the rule-based strategy is shortest due to the easy implementation. The proposed strategy invokes the rules only according to the current vehicle information, as demonstrated in Fig. 10, and the rules need to be switched according to the external inputs, therefore the duration of each step become slight longer, compared with the rule-based strategy. However, it is still suited for online application.

Table X Equivalent hydrogen consumption of batteries degradation

\begin{tabular}{lll}
\hline & $\begin{array}{l}\text { Hydrogen consumption of fuel cell } \\
\text { degradation }(\mathrm{g})\end{array}$ & $\begin{array}{l}\text { Hydrogen consumption of battery } \\
\text { degradation }(\mathrm{g})\end{array}$ \\
\hline Rule-based & 2638.3432 & 206.0461 \\
& $\mathbf{+ 8 . 9 4 \%}$ & $-34.30 \%$ \\
PMP-based & 2416.9198 & 223.4725 \\
& $-0.20 \%$ & $-28.74 \%$ \\
Rule learning-based & 2421.868 & 313.6151 \\
\hline
\end{tabular}

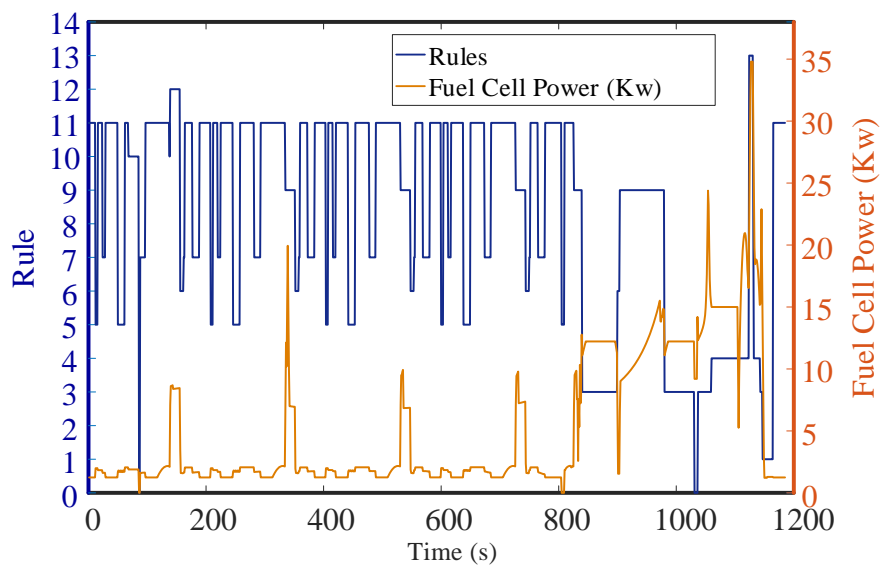

Fig. 10. Rules switching.

Table XI Calculation time with different strategies

\begin{tabular}{lll}
\hline & Total time (s) & Average time per step (s) \\
\hline Rule-based & 0.035863 & $3.026 \mathrm{e}-5$ \\
PMP-based & 0.461917 & $3.898 \mathrm{e}-4$ \\
Rule learning-based & 0.276649 & $2.335 \mathrm{e}-4$ \\
\hline
\end{tabular}

\section{CONCLUSION}

This paper proposes a multi-objective energy management strategy based on rule learning that simultaneously considers the fuel economy and power source lifetime. First, the global optimization strategy and K-means algorithm are integrated to obtain the optimal data under a specially designed blended driving cycle. Then, the improved repeated incremental pruning to produce error reduction algorithm is applied to extract rules from the optimal data set. The simulation validations were conducted based on different strategies, 
and the results manifest that the proposed algorithm can effectively improve the hydrogen consumption economy, extend the fuel cell lifetime, and furthermore highlight the potential for real-time application.

\section{ACKNOWLEDGEMENTS}

This work was supported in part by the National Natural Science Foundation of China (No. 51775063 and No. 61763021), in part by the National Key R\&D Program of China (2018YFB0105402), and in part by the EU-funded Marie Skłodowska-Curie Individual Fellowships Project under Grant 845102-HOEMEV-H2020MSCA-IF-2018.

\section{REFERENCE}

[1] H. Fathabadi, "Combining a proton exchange membrane fuel cell (PEMFC) stack with a Li-ion battery to supply the power needs of a hybrid electric vehicle," Renewable Energy, vol. 130, pp. 714-724, 2019.

[2] D. Zhou, A. Ravey, A. Al-Durra, and F. Gao, "A comparative study of extremum seeking methods applied to online energy management strategy of fuel cell hybrid electric vehicles," Energy Conversion and Management, vol. 151, pp. 778-790, 2017.

[3] L. Olatomiwa, S. Mekhilef, M. S. Ismail, and M. Moghavvemi, "Energy management strategies in hybrid renewable energy systems: A review," Renewable and Sustainable Energy Reviews, vol. 62, pp. 821-835, 2016.

[4] J. P. Trovão, P. G. Pereirinha, H. M. Jorge, and C. H. Antunes, "A multi-level energy management system for multi-source electric vehicles - An integrated rule-based meta-heuristic approach," Applied Energy, vol. 105, pp. 304-318, 2013.

[5] H. Hemi, J. Ghouili, and A. Cheriti, "A real time fuzzy logic power management strategy for a fuel cell vehicle," Energy Conversion and Management, vol. 80, pp. 63-70, 2014.

[6] J. Peng, H. He, and R. Xiong, "Rule based energy management strategy for a series-parallel plug-in hybrid electric bus optimized by dynamic programming," Applied Energy, vol. 185, pp. 1633-1643, 2017.

[7] S. Zhang and R. Xiong, "Adaptive energy management of a plug-in hybrid electric vehicle based on driving pattern recognition and dynamic programming," Applied Energy, vol. 155, pp. 68-78, 2015.

[8] S. Xie, X. Hu, Z. Xin, and J. Brighton, "Pontryagin's Minimum Principle based model predictive control of energy management for a plug-in hybrid electric bus," Applied Energy, vol. 236, pp. 893-905, 2019.

[9] Z. Hu et al., "Multi-objective energy management optimization and parameter sizing for proton exchange membrane hybrid fuel cell vehicles," Energy Conversion and Management, vol. 129, pp. 108-121, 2016.

[10] F. Odeim, J. Roes, L. Wülbeck, and A. Heinzel, "Power management optimization of fuel cell/battery hybrid vehicles with experimental validation," Journal of Power Sources, vol. 252, pp. 333-343, 2014.

[11] M. Ansarey, M. Shariat Panahi, H. Ziarati, and M. Mahjoob, "Optimal energy management in a dual-storage fuel-cell hybrid vehicle using multi-dimensional dynamic programming," Journal of Power Sources, vol. 250, pp. 359-371, 2014.

[12] L. Xu, C. D. Mueller, J. Li, M. Ouyang, and Z. Hu, "Multi-objective component sizing based on optimal energy management strategy of fuel cell electric vehicles," Applied Energy, vol. 157, pp. 664-674, 2015.

[13] W. Zhou, L. Yang, Y. Cai, and T. Ying, "Dynamic programming for new energy vehicles based on their work modes Part II: Fuel cell electric vehicles," Journal of Power Sources, vol. 407, pp. 92-104, 2018.

[14] C. H. Zheng, G. Q. Xu, Y. I. Park, W. S. Lim, and S. W. Cha, "Prolonging fuel cell stack lifetime based on Pontryagin's Minimum Principle in fuel cell hybrid vehicles and its economic influence evaluation," Journal of Power Sources, vol. 248, pp. 533-544, 2014.

[15] C. Liu and L. Liu, "Optimal power source sizing of fuel cell hybrid vehicles based on Pontryagin's minimum principle," International Journal of Hydrogen Energy, vol. 40, no. 26, pp. 8454-8464, 2015.

[16] R. Xiong, J. Cao, and Q. Yu, "Reinforcement learning-based real-time power management for hybrid energy storage system in the plug-in hybrid electric vehicle," Applied Energy, vol. 211, pp. 538-548, 2018.

[17] T. Liu, Y. Zou, D. Liu, and F. Sun, "Reinforcement Learning of Adaptive Energy Management With Transition Probability for a Hybrid Electric Tracked Vehicle," IEEE Transactions on Industrial Electronics, vol. 62, no. 12, pp. 7837-7846, 2015.

[18] B. Xu et al., "Parametric study on reinforcement learning optimized energy management strategy for a hybrid electric vehicle," Applied Energy, vol. 259, p. 114200, 2020.

[19] Y. Zou, T. Liu, D. Liu, and F. Sun, "Reinforcement learning-based real-time energy management for a hybrid tracked vehicle," Applied Energy, vol. 171, pp. 372-382, 2016.

[20] H. Liu, X. Li, W. Wang, L. Han, and C. Xiang, "Markov velocity predictor and radial basis function neural network-based realtime energy management strategy for plug-in hybrid electric vehicles," Energy, vol. 152, 2018. 
[21] Y. Zhou, A. Ravey, and M.-C. Péra, "Multi-objective energy management for fuel cell electric vehicles using online-learning enhanced Markov speed predictor," Energy Conversion and Management, vol. 213, p. 112821, 2020.

[22] J. Yuan, L. Yang, and Q. Chen, "Intelligent energy management strategy based on hierarchical approximate global optimization for plug-in fuel cell hybrid electric vehicles," International Journal of Hydrogen Energy, vol. 43, no. 16, pp. 8063-8078, 2018.

[23] S. Xie, X. Hu, S. Qi, and K. Lang, "An artificial neural network-enhanced energy management strategy for plug-in hybrid electric vehicles," Energy, vol. 163, pp. 837-848, 2018.

[24] P. M. Muñoz, G. Correa, M. E. Gaudiano, and D. Fernández, "Energy management control design for fuel cell hybrid electric vehicles using neural networks," International Journal of Hydrogen Energy, vol. 42, no. 48, pp. 28932-28944, 2017.

[25] T. Liu, X. Tang, H. Wang, H. Yu, and X. Hu, "Adaptive Hierarchical Energy Management Design for a Plug-In Hybrid Electric Vehicle," IEEE Transactions on Vehicular Technology, vol. 68, no. 12, pp. 11513-11522, 2019.

[26] L. Guo, B. Gao, Y. Gao, and H. Chen, "Optimal Energy Management for HEVs in Eco-Driving Applications Using Bi-Level MPC," IEEE Transactions on Intelligent Transportation Systems, vol. 18, no. 8, pp. 2153-2162, 2017.

[27] J. Han, D. Kum, and Y. Park, "Synthesis of Predictive Equivalent Consumption Minimization Strategy for Hybrid Electric Vehicles Based on Closed-Form Solution of Optimal Equivalence Factor," IEEE Transactions on Vehicular Technology, vol. 66, no. 7, pp. 5604-5616, 2017.

[28] Y. Liu, J. Li, Z. Chen, D. Qin, and Y. Zhang, "Research on a multi-objective hierarchical prediction energy management strategy for range extended fuel cell vehicles," Journal of Power Sources, vol. 429, pp. 55-66, 2019.

[29] K. Ettihir, M. Higuita Cano, L. Boulon, and K. Agbossou, "Design of an adaptive EMS for fuel cell vehicles," International Journal of Hydrogen Energy, vol. 42, no. 2, pp. 1481-1489, 2017.

[30] H. Liu, S.-M. Chen, and M. Cocea, "Heuristic target class selection for advancing performance of coverage-based rule learning," Information Sciences, vol. 479, pp. 164-179, 2019.

[31] S. Asadi, "Evolutionary fuzzification of RIPPER for regression: Case study of stock prediction," Neurocomputing, vol. 331, pp. 121-137, 2019.

[32] W. W. Cohen, "Fast effective rule induction," in Twelfth International Conference on International Conference on Machine Learning, 1995.

[33] S. Asadi and J. Shahrabi, "RipMC: RIPPER for Multiclass Classification," Neurocomputing, vol. 191, pp. 19-33, 2016.

[34] O. Turksoy, U. Yilmaz, and A. Teke, "Overview Of Battery Charger Topologies In Plug-In Electric And Hybrid Electric Vehicles," presented at the 16th International Conference on Clean Energy (ICCE-2018), 2018.

[35] T. Liu, X. Hu, S. E. Li, and D. Cao, "Reinforcement Learning Optimized Look-Ahead Energy Management of a Parallel Hybrid Electric Vehicle," IEEE/ASME Transactions on Mechatronics, vol. 22, no. 4, pp. 1497-1507, 2017.

[36] O. Turksoy, U. Yilmaz, and A. Teke, "Minimizing Capacitance Value of Interleaved Power Factor Corrected Boost Converter for Battery Charger in Electric Vehicles," Elektronika ir Elektrotechnika, vol. 25, no. 5, pp. 11-17, 2019.

[37] M. İnci and Ö. Türksoy, "Review of fuel cells to grid interface: Configurations, technical challenges and trends," Journal of Cleaner Production, vol. 213, pp. 1353-1370, 2019.

[38] P. Pei, Q. Chang, and T. Tang, "A quick evaluating method for automotive fuel cell lifetime," International Journal of Hydrogen Energy, vol. 33, no. 14, pp. 3829-3836, 2008.

[39] K. Namwook, C. Sukwon, and P. Huei, "Optimal Control of Hybrid Electric Vehicles Based on Pontryagin's Minimum Principle," IEEE Transactions on Control Systems Technology, vol. 19, no. 5, pp. 1279-1287, 2011.

[40] C. H. Zheng, N. W. Kim, and S. W. Cha, "Optimal control in the power management of fuel cell hybrid vehicles," International Journal of Hydrogen Energy, vol. 37, no. 1, pp. 655-663, 2012.

[41] Z. Hong, Q. Li, Y. Han, W. Shang, Y. Zhu, and W. Chen, "An energy management strategy based on dynamic power factor for fuel cell/battery hybrid locomotive," International Journal of Hydrogen Energy, vol. 43, no. 6, pp. 3261-3272, 2018.

[42] J. Wang et al., "Cycle-life model for graphite-LiFePO4 cells," Journal of Power Sources, vol. 196, no. 8, pp. 3942-3948, 2011.

[43] C. Xiang, F. Ding, W. Wang, and W. He, "Energy management of a dual-mode power-split hybrid electric vehicle based on velocity prediction and nonlinear model predictive control," Applied Energy, vol. 189, pp. 640-653, 2017.

[44] W.-S. Lin and C.-H. Zheng, "Energy management of a fuel cell/ultracapacitor hybrid power system using an adaptive optimalcontrol method," Journal of Power Sources, vol. 196, no. 6, pp. 3280-3289, 2011.

[45] H. Hemi, J. Ghouili, and A. Cheriti, "Combination of Markov chain and optimal control solved by Pontryagin's Minimum Principle for a fuel cell/supercapacitor vehicle," Energy Conversion and Management, vol. 91, pp. 387-393, 2015.

[46] L. Xu, M. Ouyang, J. Li, F. Yang, L. Lu, and J. Hua, "Application of Pontryagin's Minimal Principle to the energy management strategy of plugin fuel cell electric vehicles," International Journal of Hydrogen Energy, vol. 38, no. 24, pp. 10104-10115, 2013.

[47] J. Heil, V. Häring, B. Marschner, and B. Stumpe, "Advantages of fuzzy k-means over k-means clustering in the classification of diffuse reflectance soil spectra: A case study with West African soils," Geoderma, vol. 337, pp. 11-21, 2019.

[48] S. Garcia, J. Luengo, J. A. Sáez, V. López, and F. Herrera, "A Survey of Discretization Techniques: Taxonomy and Empirical Analysis in Supervised Learning," IEEE Transactions on Knowledge and Data Engineering, vol. 25, no. 4, pp. 734-750, 2013.

[49] S. Nataj and S. H. Lui, "Superlinear convergence of nonlinear conjugate gradient method and scaled memoryless BFGS method based on assumptions about the initial point," Applied Mathematics and Computation, vol. 369, p. 124829, 2020. 